Recepción: 20 / 12 / 2018

Aceptación: 28 / 01 / 2019

(cc)

Ciencias de la salud

Publicación: 20 / 03 / 2019

Artículo de revisión

\title{
La gestión del capital humano en medicina interna y cirugía
}

\begin{abstract}
The management of human capital in internal medicine and surgery
\end{abstract}

\section{A gestão do capital humano em medicina interna e cirurgia}

\author{
Jenny E. Landazuri-Barre ${ }^{\mathrm{I}}$ \\ jelb1388@hotmail.com \\ María E. Oña-Rivas II \\ ma.eu.ona.riva@gmail.com \\ Adriano A. Alarcón-Romero III \\ dr.aalarcon17@gmail.com \\ Luis G. Porras-Castellano IV \\ german110990@hotmail.com \\ Jhenny L. Castellano-Castellano V \\ jhennycastellano@gmail.com \\ Fabian G. Castellano-Castellano VI \\ fafiano16@gmail.com
}

Correspondencia: jelb1388@hotmail.com

\footnotetext{
I. Médico Residente de Otorrinolaringología y Cirugía Estética Facial en Otorrinos Pichincha, Ecuador.

II. Médico Residente Área de Emergencia; Hospital IESS; Portoviejo, Ecuador.

III. Médico Residente del Área de Emergencia; Hospital IESS; Portoviejo, Ecuador.

IV. Médico General; Médico de Seguridad y Salud Ocupacional Río Verde Seragroforest. S.A; Quito, Ecuador.

V. Médico General; Medico de Emergencias Centro de Salud tipo C Augusto Egas; Santo Domingo, Ecuador.

VI. Especialista en Primer Grado de Medicina General Integral; Ecuador.
} 
Jenny E. Landazuri-Barre; María E. Oña-Rivas; Adriano A. Alarcón-Romero; Luis G. Porras-Castellano; Jhenny L. Castellano-Castellano; Fabian G. Castellano-Castellano

\section{Resumen}

El capital humano se refiere a los conocimientos que ha adquirido el sujeto a través de su educación y su capacidad de poner en práctica sus habilidades y productividad, desde procesos mentales hasta los psicomotores. Los médicos, abogados, ingenieros y economistas invierten muchos años en su educación formal y en su formación en el trabajo. Gastan importantes sumas de dinero en matrículas, en formación universitaria, post grado, doctorado con el objetivo de obtener un buen salario, es decir, un rendimiento de su inversión en capital humano, que los hace especialista en su trabajo. El capital humano posee tres componentes:

La capacidad temprana o cualidades con las que un individuo cuenta, pueden ser adquiridas o innatas, las cualificaciones y conocimiento obtenidos en la educación formal y las habilidades, competencias y conocimiento conseguidos a través de la capacitación en el trabajo. El desempeño de las organizaciones de salud está parcialmente sustentado en el stock de capital humano. La medicina interna es una ciencia clínica, central a las especialidades de la medicina y la cirugía que se ocupa de la salud y enfermedad en adolescentes y adultos. En las salas de urgencias se espera que los internistas asuman la mayor autoridad y responsabilidad ante pacientes que requieren una respuesta urgente. En ocasiones este hecho crea fricciones entre el médico internista con otros colegas como por ejemplo las especialidades quirúrgicas. En el caso de los cirujanos su rol queda delimitado al tratamiento quirúrgico y seguimiento post operatorio. La metodología usada es descriptiva, con un enfoque documental, es decir, revisar fuentes disponibles en la red, como google académico, con contenido oportuno y relevante desde el punto de vista científico.

Palabras claves: Capital Humano; Inversión; Medicina Interna; Cirugía; Formación del Recurso Humano; Capital Intangible; Política de Salud.

\section{Abstract}

The early capacity or qualities with which an individual counts can be acquired or innate, the qualifications and knowledge obtained in formal education and the skills, competences and knowledge gained through on-the-job training. The performance of health organizations is partially based on the stock of human capital. Internal medicine is a clinical science, central to the specialties of medicine and surgery that deals with health and illness in adolescents and adults. In emergency rooms, internists are expected to assume the greatest authority and responsibility to patients who 
require an urgent response. Sometimes this fact creates friction between the internist doctor with other colleagues such as surgical specialties. In the case of surgeons, their role is limited to surgical treatment and postoperative monitoring. The methodology used is descriptive, with a documentary approach, that is, to review sources available on the web, such as academic google, with timely and relevant content from the scientific point of view.

Keys words: Human Capital; Investment; Internal Medicine; Surgery; Training of Human Resources; Intangible Capital; Health Policy.

\section{Resumo.}

A capacidade ou qualidades iniciais com as quais um indivíduo conta podem ser adquiridas ou inatas, as qualificações e conhecimentos obtidos na educação formal e as habilidades, competências e conhecimentos adquiridos através do treinamento no trabalho. $\mathrm{O}$ desempenho das organizações de saúde é parcialmente baseado no estoque de capital humano. A medicina interna é uma ciência clínica, central para as especialidades da medicina e cirurgia que lida com saúde e doença em adolescentes e adultos. Nas salas de emergência, espera-se que os internistas assumam a maior autoridade e responsabilidade para os pacientes que precisam de uma resposta urgente. Às vezes, esse fato cria atrito entre o médico internista e outros colegas, como especialidades cirúrgicas. No caso dos cirurgiões, seu papel é limitado ao tratamento cirúrgico e acompanhamento pós-operatório. A metodologia utilizada é descritiva, com uma abordagem documental, ou seja, para revisar fontes disponíveis na web, como o google acadêmico, com conteúdo oportuno e relevante do ponto de vista científico.

Palavras chaves: Capital humano; Investimento; Medicina Interna; Cirurgia; Treinamento de Recursos Humanos; Capital intangível; Polícia da saúde.

\section{Introducción.}

En la sociedad del siglo XXI, el culto al dinero y al consumo, la atracción por la alta tecnología, el énfasis en lo inmediato y eficaz hacen que la relación médico paciente sea un intercambio frio y distante. En medicina, el humanismo es un componente muy necesario en la relación médico - 
Jenny E. Landazuri-Barre; María E. Oña-Rivas; Adriano A. Alarcón-Romero; Luis G. Porras-Castellano; Jhenny L. Castellano-Castellano; Fabian G. Castellano-Castellano

paciente, un arte de palabras, sentimientos y actitudes. El médico internista lo expresa con tacto, compasión, optimismo y confianza.

Con el propósito de mejorar la calidad de la atención médica y disminuir costos, se requiere mayor eficiencia en la gestión de recursos intangibles como el capital humano tanto a nivel individual como organizacional. Esto no sólo beneficia al médico, centro de salud sino también a la sociedad en general porque una población más instruida es sinónimo de crecimiento económico a corto plazo y mejora en la calidad de vida de sus habitantes.

Las personas gastan en educación no solo buscando una satisfacción presente sino en miras de un mayor rendimiento en el futuro, si invierte en educación, en el futuro puedes obtener mayores ganancias de lo que invirtieron. Esta premisa también califica para el área de la salud, mientras más estudio tenga un médico, mayores herramientas posee para sanar a su paciente y precisar el diagnóstico, mejora su gestión.

Algunas de las grandes diferencias salariales de la vida diaria se deben a la existencia de calidad o excelencia entre los puestos de trabajo. Se espera que a mayor estudio mayor será el ingreso futuro, sin embargo no siempre ocurre esto, algunas autoridades en materia económica con la implementación de bajos salarios ocasionan migraciones desde el sector público de salud al privado o incluso de ser muy grande la brecha salarial entre varios países puede ocasionar migraciones a esos países cuyos salarios y beneficios sean mayores. Esto ocasiona pérdidas importantes a esa sociedad tanto en riqueza humana como material. 


\section{Metodología.}

Esta investigación está dirigida al estudio del tema Gestión del capital humano en medicina interna y cirugía. Para realizarlo se usó una metodología descriptiva, con un enfoque documental, es decir, revisar fuentes disponibles en la red, como google académico, con contenido oportuno y relevante desde el punto de vista científico para dar respuesta a lo tratado en el presente artículo y que sirvan de inspiración para realizar otros proyectos. Las mismas pueden ser estudiadas al final, en la bibliografía.

\section{Resultados.}

El capital humano se refiere a las competencias valiosas y distintivas que posee un individuo, con ellas contribuye a crear valor en el lugar de trabajo o centro de salud. No siempre obedece a un don o talento natural, en muchos casos se estudia e invierte para capacitar y actualizar los conocimientos en un área, buscando la excelencia. El capital humano se define como cantidad de conocimientos técnicos y cualificaciones que posee la población trabajadora de un país procedente de la inversión en educación formal y capacitación en el trabajo (Samuelson, 1996).

Para Nonaka, Toyama los países y las organizaciones deben integrar el conocimiento como una ventaja competitiva, si se acumula suficiente conocimiento entre los ciudadanos de un país, este tendrá un alto capital intelectual, con lo cual podrá evolucionar. La inteligencia es concebida por Sternberg y O'hara como la forma de llevar a la práctica lo que se sabe, es decir, contextualizar el conocimiento, no se requiere que la población tenga excelentes habilidades cognitivas sino que sepa aplicar lo que ya conoce (Tovar, 2017).

El capital humano posee tres componentes: 
Jenny E. Landazuri-Barre; María E. Oña-Rivas; Adriano A. Alarcón-Romero; Luis G. Porras-Castellano; Jhenny L. Castellano-Castellano; Fabian G. Castellano-Castellano

- La capacidad temprana o cualidades con las que un individuo cuenta, pueden ser adquiridas o innatas.

- Las cualificaciones y conocimiento obtenidos en la educación formal.

- Las habilidades, competencias y conocimiento conseguidos a través de la capacitación en el trabajo.

Para Gary Becker en su trabajo llamado "Human Capital", propone que las personas gastan en educación no solo buscando una satisfacción presente sino en miras de un mayor rendimiento en el futuro, si invierte en educación, en el futuro puedes obtener mayores ganancias de lo que invirtieron. Un organismo como el Gobierno, empresa o sindicato ofrecen entrenar a sus empleados con el propósito de lograr mayores utilidades (Tovar, 2017).

Figura 1. Desarrollo del Capital Humano

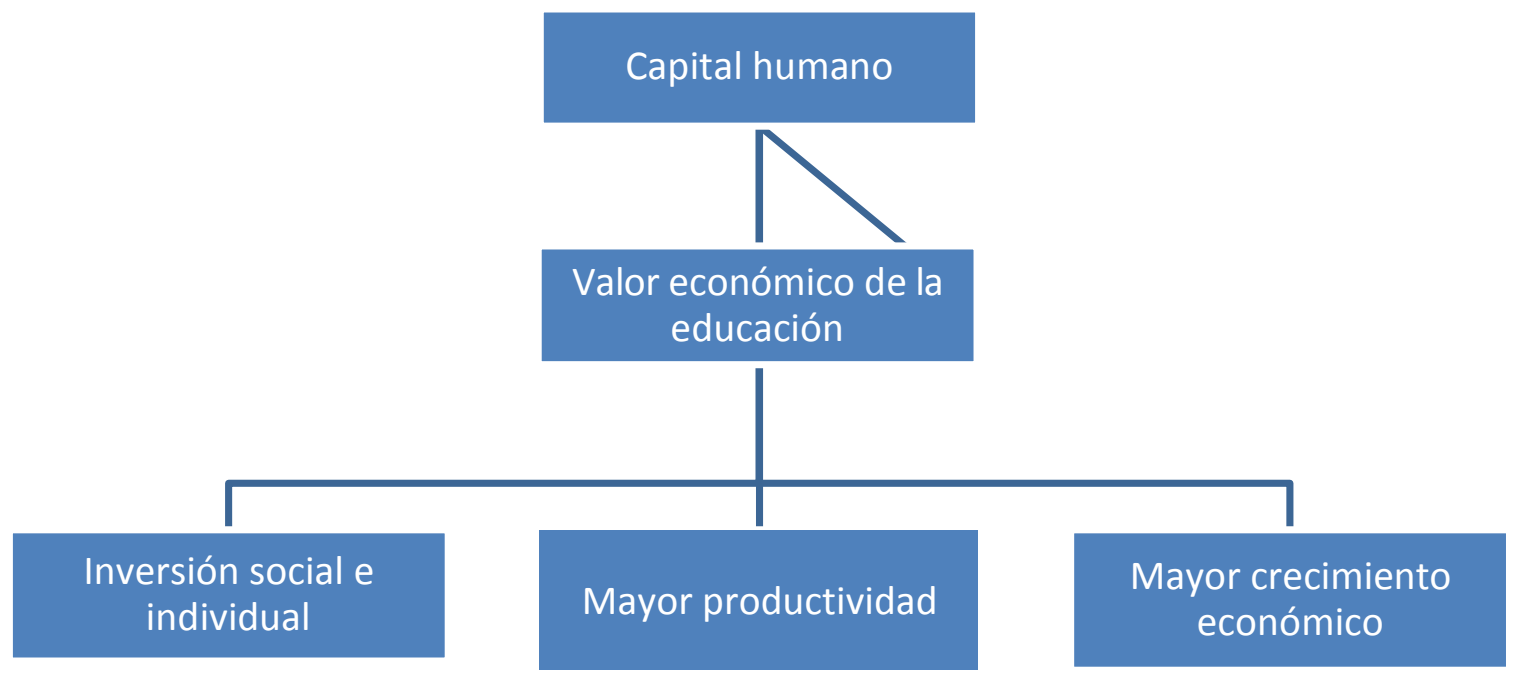

Fuente: Tovar, 2017

El entrenamiento que ofrecen las empresas se divide en dos tipos: 
- El general: es aquel del cual se obtienen resultados generales, por lo tanto, las empresas pueden hacer uso de esa capacitación que proveyeron a su personal. Por ejemplo, cuando una empresa paga a sus empleados por post grado en una universidad de prestigio. El beneficio principal se lo lleva el trabajador, si este abandona el empleo, la empresa pierde el costo de capacitación y productividad.

- El específico: sólo puede ser usado por la empresa u organización que pagó por la capacitación. Por ejemplo, la capacitación que recibe un empleado para poder realizar bien su trabajo, ya sea usar una máquina o software específico.

Los barrenderos ganan menos que los abogados cuyo empleo es más gratificante y con mejor remuneración. Sin embargo, ambos empleos son necesarios en una sociedad, no importa lo desarrollada que sea. Una de las claves a considerar de las disparidades salariales se encuentra en las diferencias cualitativas que existen entre los individuos y se atribuyen a diferente capacidad mental y física innata, de educación, formación y experiencia.

Los médicos, abogados, ingenieros y economistas invierten muchos años en su educación formal y en su formación en el trabajo. Gastan importantes sumas de dinero en matrículas, en formación universitaria, post grado, doctorado con el objetivo de obtener un buen salario, es decir, un rendimiento de su inversión en capital humano, que los hace especialista en su trabajo.

Esa inversión en capital humano no es responsabilidad únicamente del profesional de salud, también los hospitales tanto públicos como privados, el Ministerio de Salud deben planificar y vigilar el número de doctores y especialidades requeridas para brindar una atención al paciente con calidad. La planificación del recurso humano es el proceso de revisar sistemáticamente la necesidad del 
Jenny E. Landazuri-Barre; María E. Oña-Rivas; Adriano A. Alarcón-Romero; Luis G. Porras-Castellano; Jhenny L. Castellano-Castellano; Fabian G. Castellano-Castellano

recurso humano con el fin de asegurar que el número de trabajadores tenga las competencias requeridas y estén disponibles cuando se necesiten.

Tiene dos componentes o aristas: requerimiento y disponibilidad, se deriva de la Política Nacional de Salud, de las demandas actuales y futuras, del crecimiento poblacional, las migraciones, defunciones, los avances tecnológicos y cambios epidemiológicos de la salud en el país. El proceso de planificación es continuo y a largo plazo. A continuación se muestra un gráfico que precisa las diferentes áreas de acción (Castillo, 2010, pág. 11)

\section{Figura $N^{\circ}$ 2. Gestión del Recurso Humano}

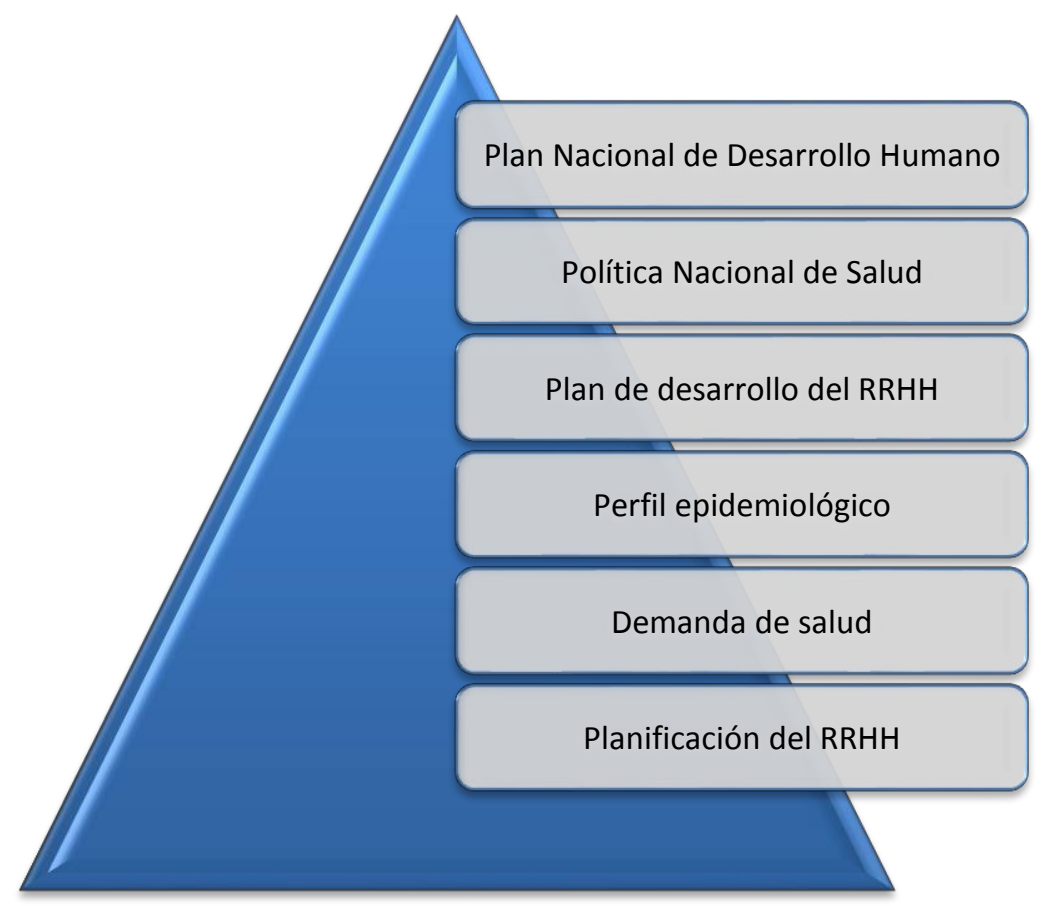

Fuente: Reyna Castillo. 2010. 
La Organización Panamericana de la Salud (PAHO) propone algunas directrices en materia de Fortalecimiento del recurso humano en salud pública, que explica muy bien la tarea pendiente de diferentes organismos e individuos del área de salud, se resumen a continuación (PAHO, 2004):

- Definir política y regulación de recursos humanos en salud: pasa por la planificación, regulación del ejercicio profesional, evaluar y supervisar la calidad de la educación y los procesos de acreditación.

- Desarrollo de la fuerza de trabajo en salud pública: planes y acciones concretas de capacitación de los profesionales de salud para el sistema de salud pública, alianzas con escuelas o programas de postgrado.

- Gestión del trabajo en salud: sistema de evaluación de desempeño, incentivos por rendimiento o productividad, manejo de conflictividad.

- Articulación de la formación y la educación de los mercados laborales: reorientación del sistema de salud hacia la atención primaria, estrategias de reclutamiento y retención, procesos de educación permanente, profesionalización del personal auxiliar.

- Reorientación de los campos y mercados laborales hacia el cambio en el sistema de salud y los modelos de atención y gestión.

El desempeño de las organizaciones de salud está parcialmente sustentado en el stock de capital humano. Si bien es cierto que el sector salud es intensivo en conocimiento, distribuido en la capacitación de los individuos, reflejado en los expedientes, bases de datos, reportes, incluidos en comités y organizaciones tanto públicas como privadas, son insuficientes los estudios que acreditan al capital humano como recurso intangible para un desempeño superior. 
Jenny E. Landazuri-Barre; María E. Oña-Rivas; Adriano A. Alarcón-Romero; Luis G. Porras-Castellano; Jhenny L. Castellano-Castellano; Fabian G. Castellano-Castellano

Con una baja oferta de universidades disponibles, elevados costos de estudio que van más allá de una matrícula, es decir, hay que contabilizar alojamiento, alimento, ropa y material bibliográfico en el monto global a invertir, dificulta o limita la formación de profesionales de la salud. Otro hecho a considerar son las bajas remuneraciones que reciben los médicos potenciando las migraciones del personal de salud muy actual en el presente.

El escaso porcentaje presupuestario asignado por los Gobiernos al sector salud contribuye a la persistencia de esta situación. La carencia de incentivos hacia aquellos profesionales con mayor grado académico o producción científica, la imposibilidad de obtener una compensación por la dedicación al trabajo de investigación, son elementos que desmotivan al personal de salud (Soto, 2011).

\section{La medicina interna}

De acuerdo con la american Board of Internal Medicine, la medicina interna es una ciencia clínica, central a las especialidades de la medicina y la cirugía que se ocupa de la salud y enfermedad en adolescentes y adultos (Gómez, 2004).

\section{Características de la medicina interna}

En 1897 William Osler predijo que la medicina interna seria la especialidad médica más completa, solicitada y gratificante, y así sucedió durante la mayor parte del siglo XX. Sin embargo, en la actualidad la medicina interna ha experimentado crecientes dificultades para perfilar su identidad y sus límites con el desarrollo de las especialidades médicas. Requiere la constante correlación de la ciencia clínica con las ciencias básicas y la conducta. 
El internista es un médico general, plural y distinguido, formado con aptitudes requeridas para el diagnóstico y tratamiento del hombre y adolescente enfermo. Se caracteriza por (Gómez, 2004):

- Habilidad para conseguir la información clínica veraz en la anamnesis y exploración física para obtener un diagnóstico.

- Sintetizar información para estructurar un diagnostico primero sindrómico y posteriormente específico.

- Aptitud para determinar la secuencia más apropiada de exploraciones complementarias.

- Capacidad para orientar o establecer el tratamiento más eficiente.

Estas características difíciles de conseguir, requieren un aprendizaje prolongado y esfuerzo continuo para incorporar los avances médicos y tecnológicos que se producen día a día. Los internistas generales constituyen el pilar básico en los centros de salud que tienen como objetivo atender a los pacientes que no requieran medidas diagnósticas o terapias especializadas. En hospitales de provincia donde no hay muchos médicos especialistas, los internistas son protagonistas en diferentes áreas álgidas (Gómez, 2004):

- Enfermedades con patologías multiorgánicas.

- Medicina de urgencias.

- Centros de formación y educación básica para internistas.

El internista debe especializarse en áreas concretas para diagnosticar teniendo presente la visión integral del hombre. A partir de la medicina interna muchas especialidades médicas: cardiología, neumología, oncología, infectología, cirugía, etc., acuden en consultoría o referencia. 
Jenny E. Landazuri-Barre; María E. Oña-Rivas; Adriano A. Alarcón-Romero; Luis G. Porras-Castellano; Jhenny L. Castellano-Castellano; Fabian G. Castellano-Castellano

Estar actualizado de los avances médicos y tecnológicos es valioso pero no basta para ser un buen doctor. La tecnología médica es el conjunto de medicamentos, aparatos, procedimientos médicos y quirúrgicos y sistemas organizativos con los que se presta atención medico asistencial. El acceso de los pacientes a las nuevas redes de información facilitan al enfermo consultar su sintomatología, vuelva a consulta indicándole al médico la exploración y tratamiento que necesita según lo leído, cambiando los roles de la relación médico - paciente.

La actitud más adecuada es compaginar la técnica con la empatía, el pilar fundamental que sustenta el proceso de toma de decisiones, asienta la confianza entre médico y paciente. Lograr curaciones de enfermedades en forma oportuna y sencilla sin invadir o explorar otras áreas fuera del campo del médico internista busca:

- Dar una buena atención médica, de calidad.

- Evitar el sufrimiento innecesario, teniendo como base la ética profesional.

- Remitir al especialista de ser necesario y seguir la evolución del paciente.

Otro aspecto dentro del área de la medicina interna es la medicina intensiva. En las salas de urgencias se espera que los internistas asuman la mayor autoridad y responsabilidad ante pacientes que requieren una respuesta urgente. En ocasiones este hecho crea fricciones entre el médico internista con otros colegas como por ejemplo las especialidades quirúrgicas.

Es importante destacar que mientras el enfermo no tenga una urgencia quirúrgica o un diagnóstico preciso, no debe pasar a manos de otros médicos, el internista debe evaluarlo y cuando este claro el diagnóstico remitirlo a otro especialista a fin de sanarlo. En el caso de los cirujanos su rol queda delimitado al tratamiento quirúrgico y seguimiento post operatorio. 
El tipo de paciente que acude a consulta con un médico internista suele ser adulto mayor, sienten malestar y buscan respuesta para mejorar su salud, casi siempre acuden a consulta solos y cuando su salud se ha deteriorado significativamente. Es una búsqueda de medicina y/o tratamientos más que chequeos de rutina o para prevenir problemas de salud a futuro.

\section{Cirugía vs. Internista}

Los cirujanos son médicos al igual que los internistas, la diferencia estriba que el primero tiene una especialidad en cirugía. Los internistas tratan los problemas de salud con medicamentos, atiende aquellas patologías que afectan diversos órganos del cuerpo, se ocupa de enfermedades sistémicas, aglutina la función de varios especialistas y evita que los tratamientos se contrarresten entre sí. Los cirujanos tratan mediante cirugías (cortar y/o abrir diferentes partes del cuerpo) y mejorar la salud del paciente.

Los cirujanos operan a los pacientes en centros de salud, trabajan con gran habilidad manual, usando una serie de instrumentos, técnicas y equipos. Generalmente, el cirujano vera por primera vez al paciente después de haber sido remitido por el médico internista. Además de trabajar en el quirófano, los cirujanos evalúan a los pacientes en consulta médica, tiene que explicar el por qué necesita operar, que ocurrirá durante la operación, explicar riesgos y efectos secundarios, cuánto tiempo durará la operación y el tiempo de recuperación luego de ser intervenido quirúrgicamente.

Aclaradas las funciones de ambas ramas de la medicina, se puede precisar que lejos de competir el internista con el cirujano cada uno tiene su rol en los centros de salud, claramente definidos, en términos del enfoque del capital humano se espera que el cirujano perciba una remuneración mayor que un internista, posee mayor formación técnica, conocimiento y habilidades adquiridas durante su formación. 


\section{Conclusión.}

El presente artículo desarrolló el tema de la gestión del capital humano en la medicina interna y cirugía. Se define como competencias valiosas y distintivas que posee un individuo, con ellas contribuye a crear valor en el lugar de trabajo o centro de salud. No siempre obedece a un don o talento natural, en muchos casos se estudia e invierte para capacitar y actualizar los conocimientos en un área, buscando la excelencia. El capital humano se define como cantidad de conocimientos técnicos y cualificaciones que posee la población trabajadora de un país procedente de la inversión en educación formal y capacitación en el trabajo.

Dicha inversión en dinero y tiempo se realiza en el presente con el propósito de obtener un mayor rendimiento de lo invertido a futuro. Se espera que mientras mayor calificación posea un individuo mayor será la remuneración del mismo, sin embargo, esto no siempre ocurre, ocasionando migraciones en búsqueda de mejores salarios y beneficios de centros rurales a urbanos, centros públicos a privados, generando una baja en la calidad y atención oportuna de pacientes.

Otro aspecto dentro del área de la medicina interna es la medicina intensiva. En las salas de urgencias se espera que los internistas asuman la mayor autoridad y responsabilidad ante pacientes que requieren una respuesta urgente. En ocasiones este hecho crea fricciones entre el médico internista con otros colegas como por ejemplo las especialidades quirúrgicas. Mientras el enfermo no tenga una urgencia quirúrgica o un diagnóstico preciso, no debe pasar a manos de otros médicos, el internista debe evaluarlo y cuando este claro el diagnóstico remitirlo a otro especialista a fin de sanarlo. En el caso de los cirujanos su rol queda delimitado al tratamiento quirúrgico y seguimiento post operatorio. 
La inversión en capital humano no es responsabilidad únicamente del profesional de salud, también los hospitales tanto públicos como privados, el Ministerio de Salud deben planificar y vigilar el número de doctores y especialidades requeridas para brindar una atención al paciente con calidad. La planificación del recurso humano es el proceso de revisar sistemáticamente la necesidad del recurso humano con el fin de asegurar que el número de trabajadores tenga las competencias requeridas y estén disponibles cuando se necesiten. Es la mejor inversión para cualquier sociedad porque implica mejorar procesos, mayor productividad variables claves para potenciar el crecimiento económico. Ir de algo tan pequeño y puntual como capacitarse un individuo, mejora su entorno donde trabaja y gana el país.

\section{Bibliografía.}

Gómez J. (2004). La medicina interna: situación actual y nuevos horizontes. Recuperado por http: www.scielo.isciii.es

Blanco M. (2014). La medicina interna en el ambiente hospitalario. Recuperado por http: www.scielo.sid.cu

Castillo R. (2010). Manual de procedimientos, planificación y programas de recursos humanos. Recuperado por http: www.paho.org

Soto A. (2011). Recursos humanos en salud. Revista Perú Médica. Recuperado por http: www.scielo.org.pe

Samuelson P. (1997). Economía. Mc Graw Hill. Madrid.

Vidal P. (2012). Gestión de recursos humanos en triage de servicio de emergencia hospitalaria. Recuperado por http: www.fcea.edu.uy

Nigenda G. (2013). Recurso humano para la salud en el contexto de la reforma sanitaria en Méjico: formación profesional y mercado laboral. Recuperado por http: www.anmm.org.mx

Organización Panamericana de Salud. (2004). Fortalecimiento del recurso humano en salud pública. Recuperado por http: www.paho.org

Tovar B. (2017). La teoría del capital humano llevada a la práctica en las ciudades de aprendizaje. Recuperado por http: www.revistanoesis.mx 